\title{
Studies on Halo Formation in a Long Magnetic Quadrupole FODO Channel First Experimental Results
}

\author{
P-Y Beauvais ${ }^{1}$, D.Bogard ${ }^{1}$, P-A Chamouard ${ }^{1}$, R.Ferdinand ${ }^{1}$, \\ G. Haouat ${ }^{2}$, J-M Lagniel ${ }^{1}$, J-L Lemaire ${ }^{2}$, N. Pichoff ${ }^{2}$, C. Ruiz ${ }^{2}$. \\ 1) CEN SACLAY-DSM/LNS and GECA, BP 2, 91191 Gif-sur-Yvette. France. \\ 2) CEA-BIII/PTN, BP 12, 91680 Bruyères-le-Châtel. France.
}

\begin{abstract}
A study is progress to prepare an experiment on the transport of an intense proton beam, with high optical qualities, through a periodic magnetic quadrupole FODO channel (29 periods). It is intended to provide a better understanding of how halo develops and can be controlled as a crucial issue for the design of high-intensity linear accelerators. Simulations were done showing that this experiment can be performed with a high-brightness proton, beam produced by a duoplasmatron source, with an energy of $500 \mathrm{keV}$ and a current of tens of mA. Beam pulse length will be less than $1 \mathrm{~ms}$ and normalized emittance better than $1 . \pi \mathrm{mm} \cdot \mathrm{mrad}(90 \%$ of total beam).Thus, transverse tune can be sufficiently depressed in the transport channel to allow instabilities to grow and halo to develop or stay under control.

The overall experiment is divided in two phases. Phase 1, the subject of this paper, is dedicated to precise measurements on initial beam conditions, such as emittance, for the beam entering the FODO channel. Phase 2, concerning the halo formation phenomena will be carried on latter, if results of phase 1 are satisfactory.
\end{abstract}

\section{INTRODUCTION}

For many years, experimental studies on emittance growth of beams transported under space charge conditions have been done at different laboratories[1]. The aim was, and is still, to test the calculations and the simulations made for long focusing continuous channels and alternating strong focusing quadrupole channels both for electron and heavy ion beams. Of course, the cost of such transport lines strongly limits the experiments and they have to be achieved in several steps. Previous results seem to be limited by the sensitivity of the experimental setups. Therefore, more sensitive diagnostics are needed for these tests. With the growing interest for dedicated high-intensity $\mathrm{CW}$-beam linear accelerators, linacs must achieve very low beam losses to insure the maintainability of the structure. Instabilities in the beam that lead to a halo are still a real concern; so, understanding of halo formation, and controlling its development is a key issue for all designers. Recently new insights $[2,3]$ suggested that similar experiments should be performed again with better beam instrumentation. Efforts have to be put into diagnostics better adapted to the requirements of measuring the low density part of the beam included in the halo along with the optical qualities of the initial beam (emittance, profile,mismatching and missteering).

Because of budget constrainsts, a proposal was made, early in 1994, to use an existing quadrupole channel of 29 periods. This system consists of the focusing quadrupoles of the LNS 20.MeV Alvarez linac not powered with RF[3]. All the existing hardware was available and the only major modification was to update the quadrupole system. The power supplies offered all the flexibility that was necessary to cover a wide range of tuning.

\section{THEORETICAL ASPECTS - BEAM SIMULATIONS}

The FODO channel has a variable period because spacing of the quadrupoles increases along the structure due to the location of the drift tubes. Matching between adjacent cells has to be done to obtain the stable envelopes for different phase advances at zero beam current $\left(\sigma_{0}\right)$ and desired transported current $\left(\sigma_{t}\right)$. We chose $\sigma_{0}=60^{\circ}$ and $100^{\circ}$. The core envelope is optimized for a K.V distribution. Simulations are done using the particle core model (PCM) for three concentric distributions of 2000 particles each: the first distribution treats the core, the second one the border part and the third one the outer part of the beam. These test particles are uniformly distributed in the phase space and tracked along the structure. It is expected by many authors that above $\sigma_{0}=90^{\circ}$, instabilities develop and emittance grows. This is very well demonstrated in our simulations[3]. It is observed that phase space deteriorates and emittance grows with increasing beam current. The envelope

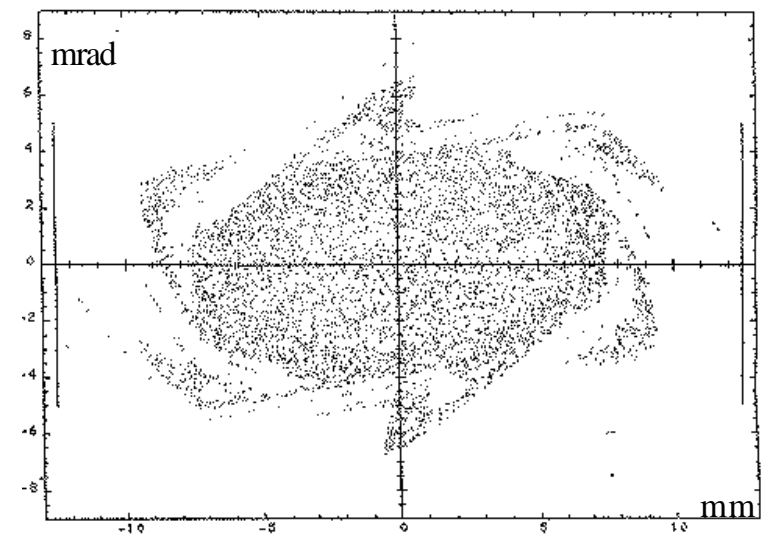

Figure 1 : Transverse phase space portrait at the end of the FODO channel. Halo which forms tails can be seen 
starts to oscillate and a halo grows around the core. Studies of these phenomena are in progress but it is beyond the scope of this paper to recall all the theoretical aspects.

\section{EXPERIMENTAL SET-UP}

The experimental set-up corresponding to phase 1 is shown in Figure 2. It consists of a duoplasmatron source housed in a high voltage terminal $\mathbf{1}$ delivering, for the purpose of the experiment, a proton beam up to $50 \mathrm{~mA}$ at $500 \mathrm{keV}$. The pulse duration is set to $500 \mu$ s to allow for transient effects to die before any measurements are performed on the beam. In this respect, we can consider that we are dealing with an equivalent DC beam for both simulation and reasurement. The intermediate section between the FODO channel 2 and the beam source holds all the instrumentation required for beam diagnostics during phase 1 . It will become the matching section for phase 2 . At present time, the only focusing element is a quadrupole triplet $\mathbf{3}$ used to form the beam image dowstream. A dipole magnet $\mathbf{4}$ allows for beam analysis: its diagnostic is put in viewing box $\mathbf{5}$. The other viewing boxes house the main beam diagnostics:

- box 6 contains a Faraday cup, collimators and a sampling hole,

- box 7 contains collimators, sampling holes, and a slit,

- box $\mathbf{8}$ contains a pepper-pot to sample the beam

- box 9 holds the instrumentation dedicated to emittance, beam profile and beam current measurements.

For phase 2 experiment, viewing box 9 will be replaced by a set of magnetic quadrupole lenses to properly match the beam to the FODO channel.

\section{EXPERIMENTAL METHODS}

\section{A.Beam Instrumentation}

Usual methods are applied for beam current and beam centroïd measurements; which are: Faraday cups, current transformers located at positions $\mathbf{6}, \mathbf{8}$ and $\mathbf{9}$ and multi-wire profile monitors, scintillating screen located at position 9. At places 6 and 7, variable apertures allow for limitation of the transverse emittance. The main effort was done to achieve a maximum sensivity on beam emittance measurements. Two methods were used for cross-checking the measurements: i) the hole-profile method (HPM) which gives good accuracy on beam brightness and on beam aberrations, ii) the pepper-pot method (PPM) where a scintillating screen gives a full video information in only one beam shot. Unfortunately, the HPM takes a long time (about 30 minutes ) since a sampling hole has to be stepped across the beam. The latter method is inspired of an imaging method widely used on the ELSA linac[4] and should be capable, for phase 2 , of measuring density distributions over at least 4 decades.

\section{B.Pepper-pot Method}

The $300-\mu \mathrm{m}$ thick graphite sampling plate with $200 \mu \mathrm{m}$ diameter holes spaced by $2 \mathrm{~mm}$ is used for the pepper-pot method. The scintillator is $64 \mathrm{~cm}$ behind. It is chromium doped alumina covered by a 300 . $\AA$ gold deposit to eliminate the charges. Beamlet spots are visualized with a CCD intensified camera. The measurement integrates over

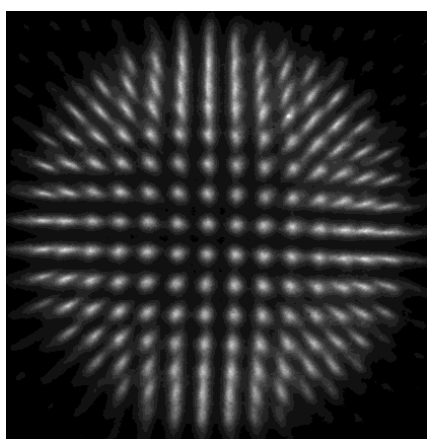

Figure 3: Pepper-pot image of beam on scintillating screen. the beam pulse duration.

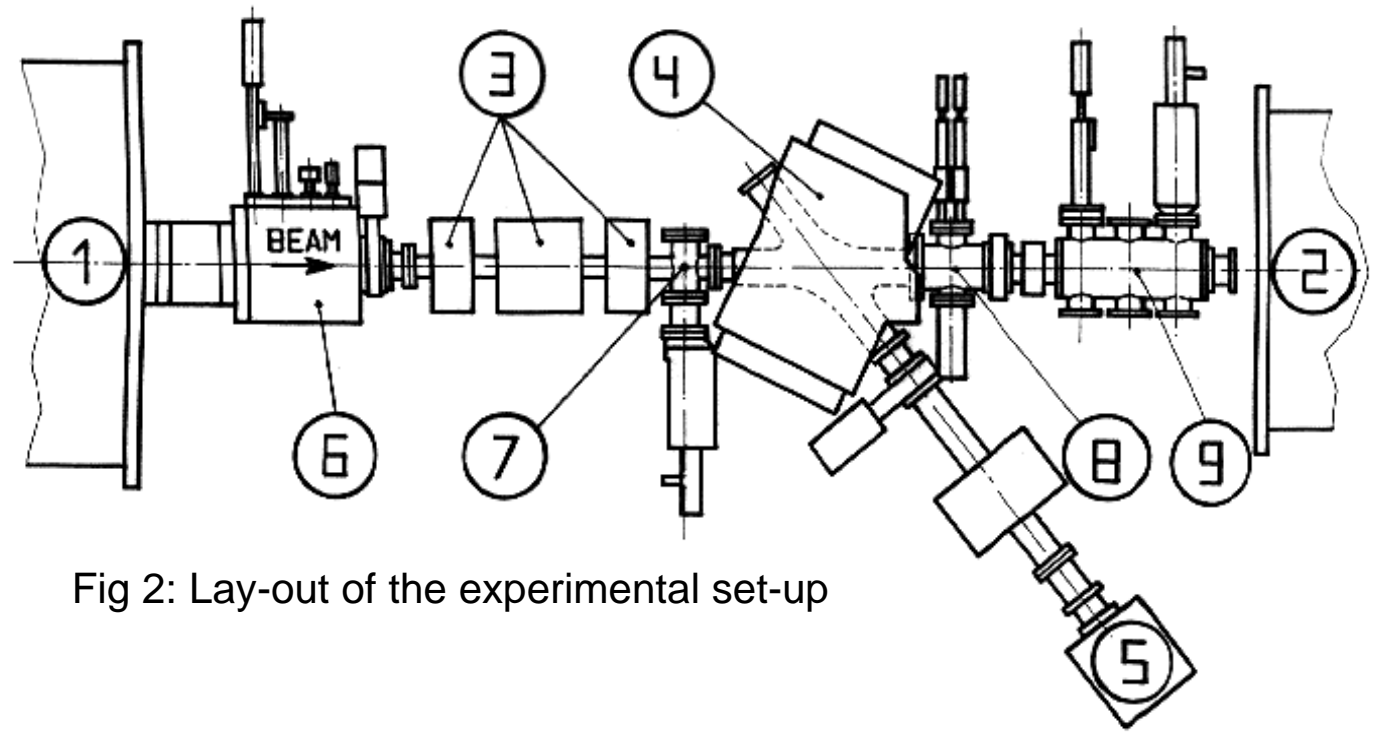




\section{Profile-Hole Method}

A multi wire profile monitor is placed $3 \mathrm{~m}$ behind a $200 * 200$ $\mu \mathrm{m}$ square sampling hole[5]. This monitor is made of 64 wires (gold covered tungsten of $30 \mu \mathrm{m}$ diameter), spaced by $300 \mu \mathrm{m}$ in the center and $500 \mu \mathrm{m}$ at the edges. A $100 \mathrm{~V}$ bias eliminates the secondary-electron emission. The sampling step size of the apparatus is $.2 \mathrm{~mm}$.

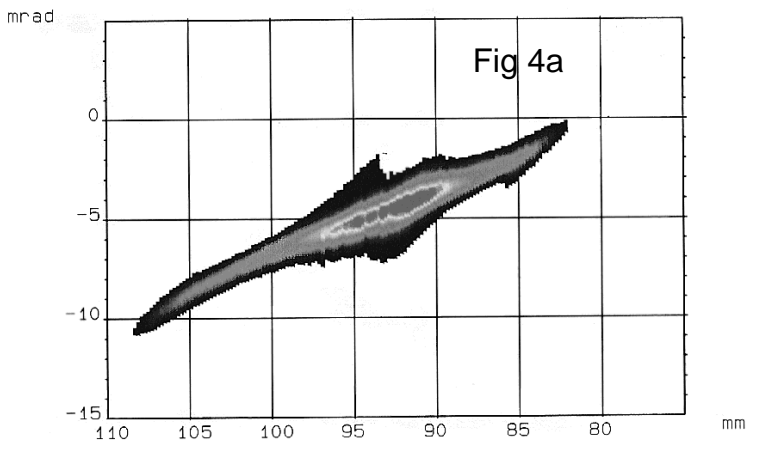

Figure 4a shows a typical emittance contour including $90 \%$ of the beam. It corresponds to a $30 \mathrm{~mA}, 500 \mathrm{keV}$ beam with a normalized emittance of $\mathrm{Sn} / \mathrm{p}=.6 \mathrm{~mm} . \mathrm{mrad}$.

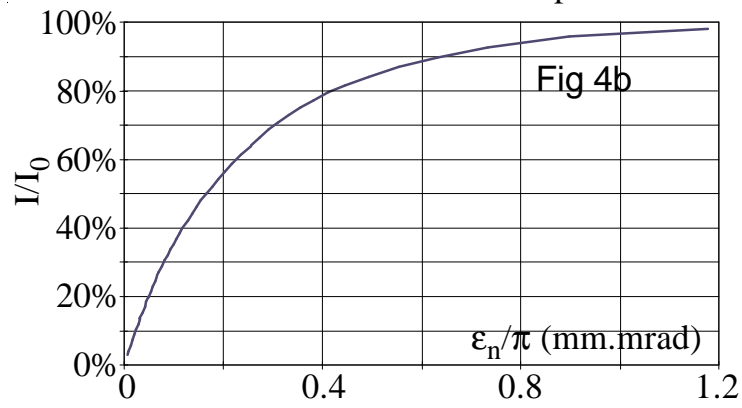

Figure $4 \mathrm{~b}$ represents the brightness curve corresponding to the emittance shown on figure $4 \mathrm{a}$. I/I0 is the relative intensity of the beam included in this emittance contour.

\section{V-. EXPERIMENTAL RESULTS}

We measured the relative amount of the different species produced by the ion source. It turned out that $\mathrm{H} 2+$ and $\mathrm{H} 3+$ represent respectively about $10 \%$ and $1 \%$ of the beam. The highest $\mathrm{H}+$ percentage was reached for $50 \mathrm{~mA}$ total beam current. For future experiments we will have to deal with these unwanted species.

Comparison of the two emittance-measurement methods was done for a typical full beam of $20 \mathrm{~mA}$. One cannot use the triplet magnet in the PPM because separation of the species produces an unacceptable background. However, without the triplet magnet, the beam was too wide on the scintillator, so it was decided to collimate the beam with a $\emptyset 4 \mathrm{~mm}$ collimator, in order to compare the emittance measurement methods ( space charge effects due to the difference between species was neglegeable).

Figure 5 shows the collimated-beam brigthness obtained by the two methods. The PPM leads to a measured brightness 2 times greater than that measured by the HPM. After having corrected the data from diffusion effects in the scintillator, we assume that this difference comes from the fact that the PPM data are integrated over the whole pulse duration even though the HPM samples during only $5 \mu \mathrm{s}$ in the stable part of the pulse. Further experiments are in progress to verify this hypothesis.

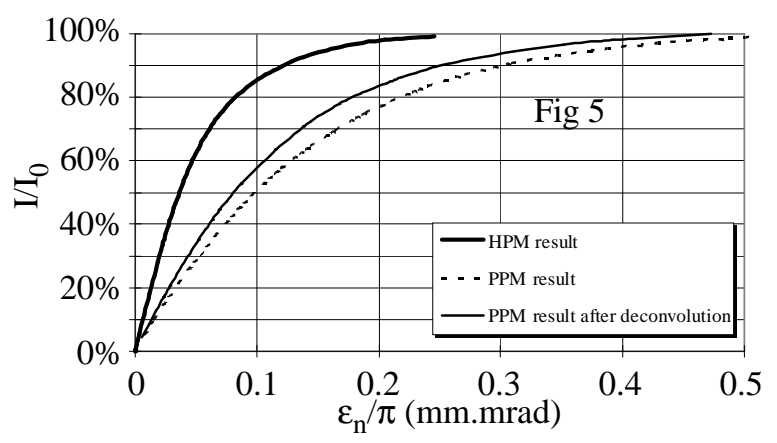

\section{CONCLUSION}

The present emittance results are good enough ( . $6 \mathrm{~mm} . \mathrm{mrad}$ for $90 \%$ of the particle of a $30 \mathrm{~mA}$ beam) to allow experiment of phase 2 to be done with a sufficiently large excursion of the tune depression.. Nevertheless some more measurements will be performed to find the best sets of source parameters which minimize the transverse emittance.

The chromium-doped alumina scintillators, which were used for the present experiment, will be replaced by faster scintillators in order to measure time-resolved profiles and emittances. These are needed to explain differences between the two experimental methods, and to allow measurements of the halo evolution during the beam pulse.

To eliminate the undesirable species in the proton beam a Wien filter has been designed. It will be installed before starting phase 2 of FODO experiment.

\section{ACKNOWLEDGEMENTS}

The authors want to express their thanks to S.Joly for encouraging and supporting the FODO experiment. It is also a pleasure to acknowledge the help of P.Hardy, S.Seguin and S.Striby

\section{REFERENCES}

[1].see for example,D.Kehne, M.Reiser and H.Rudd, "Experimental studies of emittance growth due to initial mismatch of a space-charge dominated beam in a solenoidal focusing channel", PAC91 Conf.Proc; p248.

[2] J-M Lagniel, "Chaotic behaviour induced by space charge", EPAC94, Conf.Proc, p1177

[3] J-M Lagniel, "Etude expérimentale du halo crée par la charge d'espace, proposition", LNS-SM-94-06, int.rep.

[4] G.Haouat and al., "Halo of a high-brightness electron beam", this conference

[5] P-Y.Beauvais, R.Ferdinand, J-L.Lemaire, "Mesure fine de l'émittance d'un faisceau d'ions", LNS/SM 93-08, int.rep 